\title{
The Origin and Evolution of the Black Hole Binary XTE J1118+480
}

\author{
Jonay I. González Hernández ${ }^{1,2}$, Rafael Rebolo ${ }^{1,2}$ and Jorge Casares ${ }^{1,2}$ \\ ${ }^{1}$ Instituto de Astrofísica de Canarias, Vía Láctea, s/n, E-38205 La Laguna, Tenerife, Spain \\ email: jonay@iac.es \\ ${ }^{2}$ Departamento de Astrofísica, Universidad de La Laguna, E-38206 La Laguna, Tenerife, Spain
}

\begin{abstract}
Black hole X-ray binaries with large mass ratios and short orbital periods are expected to change their orbital period due to magnetic breaking, mass loss, gravitational radiation, or mass evaporation of the black hole in alternative descriptions of gravity, like in braneworld gravity scenarios.

The black hole X-ray binary XTE J1118+480, consisting of a late-type secondary star orbiting a $\sim 8 M_{\odot}$ black hole in a 4.1-hr period, offers a unique opportunity to test these models. New spectroscopic data allow us to determine the time of the inferior conjunction of the secondary star at different epochs. Observations over a 10 year span will provide constraints on the rate of any orbital period change.

We present here a preliminary radial velocity curve obtained with the $10.4 \mathrm{~m}$ GTC telescope equipped with OSIRIS medium-resolution spectrograph, as part of an ongoing long-term program to study the orbital period evolution in this binary.
\end{abstract}

Keywords. black hole physics, techniques: radial velocities, stars: individual (XTE J1118+480), $\mathrm{X}$-rays: binaries, supernovae: general

The black hole X-ray binary XTE J1118+480 is located in Galactic halo regions and its galacto-centric motion is similar to those of halo stars (Mirabel et al. 2001). However, the high metal content of the secondary star in this system suggests that the system probably originated in the Galactic plane (González Hernández et al. 2006, 2008b), and was launched into its current location via an asymmetric kick in a supernova/hypernova explosion (Gualandris et al. 2005). The galacto-centric orbit of the system crosses the Galactic plane backwards in time in roughly 11 Myr. This particularity has been used to derive an upper-limit to the asymptotic radius curvature radius, $L \leqslant 80 \mu \mathrm{m}$, in the Anti-de-Sitter (AdS) braneworld Randall-Sundrum gravity model (Psaltis 2007).

During the last decade, we have been collecting high-quality spectroscopic data of black-hole X-ray binaries (González Hernández et al. 2004, 2008a, 2008b, 2010, 2011) and neutron-star X-ray binaries (Casares et al. 2007, 2010; González Hernández et al. 2005). In particular, medium-resolution spectroscopy has been obtained with Keck II/ESI which allowed us to derive radial velocities (RVs) of the secondary star in XTE J1118+480 (González Hernández et al. 2008b). These RV measurements give an accurate determination of the time of the inferior conjunction of the secondary star, $T_{0}$, in this system. By comparing the $T_{0}$ values obtained at different epochs, one can determine the orbital period derivative. This provides not only important information on the variation of the angular momentum of the system but also places strong constraints on the size of the extra dimensions $L$ in the context of AdS braneworld gravity theory (Johannsen 2009).

We have performed spectroscopic observations with the OSIRIS medium-resolution spectrograph, attached to the $10.4 \mathrm{~m}$ GTC telescope at the Observatorio del Roque de los Muchachos in La Palma (Spain). In Fig. 1, we display the RV measurements obtained 


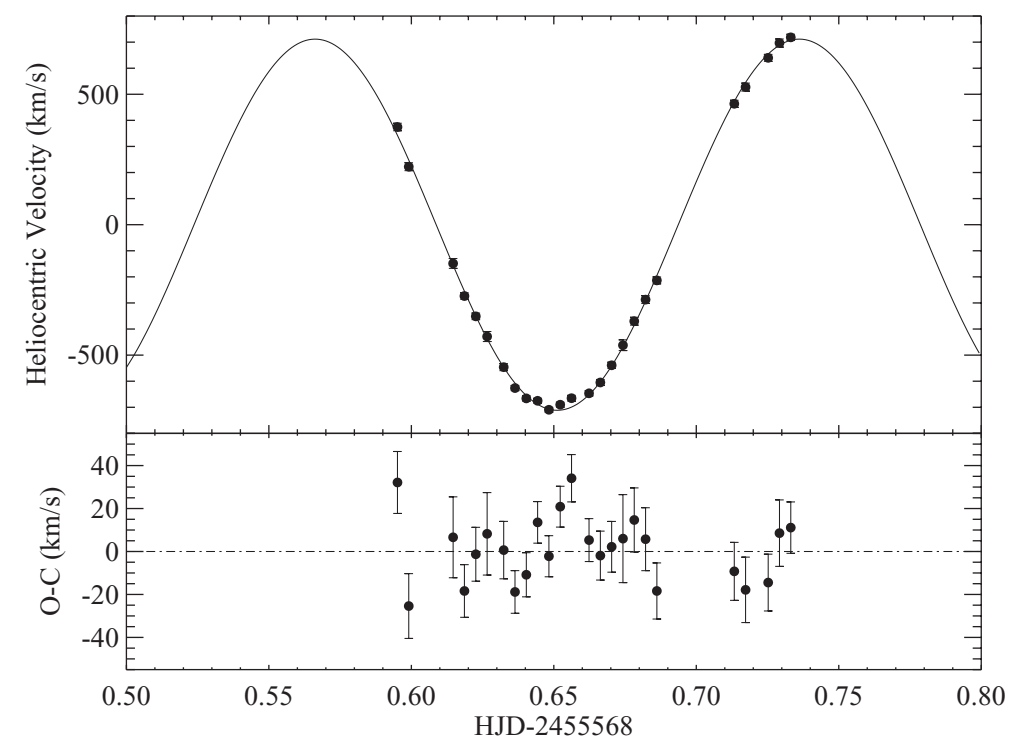

Figure 1. Top panel: radial velocities of the secondary star in the black hole X-ray binary XTE J1118+480 obtained from GTC/OSIRIS spectroscopic data taken over one night in January 2011, folded on the best-fitting orbital solution. Bottom panel: residuals of the fit, with a rms of $\sim 15 \mathrm{~km} \mathrm{~s}^{-1}$.

in one night which allow us to derive the time of the inferior conjunction of the companion star. This value of $T_{0}$ will be used, in combination with previous $T_{0}$ measurements, to derive the orbital period variation with time and the implications on the curvature radius $L$, which gives the rate at which the black hole evaporates in the AdS braneworld gravity.

\section{References}

Casares, J., Bonifacio, P., González Hernández, J. I., et al., 2007, A\&\&A, 470, 1033

Casares, J., González Hernández, J. I., Israelian, G., \& Rebolo, R. 2010, MNRAS, 401, 2517

González Hernández, J. I., Rebolo, R., Israelian, G., et al., 2004, ApJ, 609, 988

González Hernández, J. I., Rebolo, R., Israelian, G., et al., 2005, ApJ, 630, 495

González Hernández, J. I., Rebolo, R., Israelian, G. et al., 2006, ApJ, 644, L49

González Hernández, J. I., Rebolo, R., \& Israelian, G. 2008a, A $6 A$, 478, 203

González Hernández, J. I., Rebolo, R., Israelian, G., et al., 2008b, ApJ, 679, 732

González Hernández, J. I. \& Casares, J. 2010, A $\mho \mho A, 516$, A58

González Hernández, J. I., Casares, J., Rebolo, R., et al., 2011, arXiv:1106.4278

Gualandris, A., Colpi, M., Portegies Zwart, S., \& Possenti, A. 2005, ApJ, 618, 845

Johannsen, T. 2009, A\&A, 507, 617

Psaltis, D. 2007, Phys. Rev. Lett., 98, 181101

Mirabel, I. F., Dhawan, V., Mignani, R. P., et al., 2001, Nature, 413, 139 